\title{
AVALIAÇÃO DE HABILIDADES ORAIS DE CRIANÇAS NASCIDAS PRÉ-TERMO
}

\section{Assessing oral skills of preterm infants}

\author{
Aneline Maria Ruedell ${ }^{(1)}$, Leris Saleti Bonfanti Haeffner ${ }^{(2)}$, Lisiane Martins Silveira ${ }^{(3)}$, \\ Márcia Keske-Soares ${ }^{(4)}$, Angela Regina Maciel Weinmann (5)
}

\begin{abstract}
RESUMO
Objetivo: avaliar as habilidades orais de crianças nascidas pré-termo aos 4 e 6 meses de idade corrigida. Método: foram avaliadas 14 crianças, nascidas com idade gestacional entre 29 e 35 semanas, no Ambulatório de Seguimento de Prematuros do Hospital Universitário de Santa Maria, no estado do Rio Grande do Sul. A avaliação aos 4 e 6 meses de idade corrigida compreendeu movimentos de língua, lábios e mandíbula durante a utilização da mamadeira ou seio, sendo que aos 6 meses também foi avaliado a retirada do alimento pastoso da colher, o uso do copo, e a mascagem com bolacha. Todas as avaliações foram filmadas e analisadas por 3 fonoaudiólogas. Um questionário sobre os hábitos alimentares das crianças foi respondido pelos pais. Resultados: aos 4 e 6 meses o vedamento labial adequado foi observado em $71,4 \%$ e 85,7\%, respectivamente. Aos 6 meses em $78,6 \%$ das crianças o lábio superior retirou de forma eficiente o alimento pastoso da colher, $42,9 \%$ conseguiram sorver o líquido do copo de forma adequada e $57,1 \%$ tiveram dificuldade no uso do copo. Conclusão: neste estudo, as crianças que nasceram prematuras aos 4 e 6 meses de idade corrigida apresentaram desenvolvimento das habilidades orais em uma seqüência progressiva, no entanto apresentaram comprometimento do vedamento labial durante a sucção e dificuldades no uso da colher e do copo para sua alimentação.
\end{abstract}

Descritores: Criança; Métodos de Alimentação; Prematuro

(1) Professora Assistente da Universidade Estadual do Oeste do Paraná (UNIOSTE), Cascavel, Paraná, Brasil; Mestre em Distúrbios da Comunicação Humana pela Universidade Federal de Santa Maria.

(2) Médica Pediatra; Professora Associada do Departamento de Pediatra e Puericultura da Universidade Federal de Santa Maria; Doutora pela Faculdade de Medicina de Ribeirão Preto, Universidade de São Paulo (FMRP-USP).

(3) Fonoaudióloga, Mestranda do Programa Distúrbios da Comunicação Humana pela Universidade Federal de Santa Maria.

(4) Fonoaudióloga, Professor Adjunto de Departamento de Fonoaudiologia da Universidade Federal de Santa Maria; Doutora em Linguística Aplicada pela Pontifícia Universidade Católica do Rio Grande do Sul (PUC-RS).

(5) Médica Pediatra e Neonatologista; Professor Associado do Departamento de Pediatra e Puericultura e do Programa de Pós-Graduação em Distúrbios da Comunicação Humana da Universidade Federal de Santa Maria; Doutora pela Faculdade de Medicina de Ribeirão Preto, Universidade de São Paulo (FMRP-USP).

Conflito de interesses: inexistente

\section{INTRODUÇÃO}

Ao nascimento e nos primeiros meses de vida, a criança apresenta reflexos orais importantes para a sua alimentação que, com o desenvolvimento do sistema nervoso central, são substituídos por movimentos voluntários normais ${ }^{1}$. O reflexo e após o movimento voluntário de sucção, quando realizado de forma adequada, permite a contenção do leite na cavidade oral e o início do processo alimentar. Contudo, para que isso ocorra, é necessário haver uma organização na atividade muscular da mandíbula, lábios e língua, além de força de sucção suficiente que possibilite a expressão do leite ${ }^{2}$.

A partir do $4^{\circ}$ ou do $6^{\circ}$ mês de vida, tem sido recomendado a introdução de outros alimentos, oferecidos com a colher, com o copo ou diretamente na boca do bebê. Na habilidade para o uso da colher, observa-se que o lábio superior procura 
abaixar e auxiliar na retirada do alimento, já para o uso do copo, ocorre o padrão habitual da sucção, sendo que quando o líquido chega à boca, a criança excursionará e estabilizará a mandíbula e a língua, projetando-a antes da deglutição $0^{3,4}$. Na introdução de alimentos pastosos, a criança utilizará a sucção e movimentos de abertura e fechamento da boca e, quando o alimento for colocado na posição lateral da boca, iniciarão os primeiros movimentos mastigatórios ${ }^{3}$.

A avaliação das habilidades orais de crianças prematuras ainda tem sido pouco estudada, mas o conhecimento sobre o assunto tem extrema importância, uma vez que pode levar a uma intervenção mais precoce evitando-se, assim, problemas nutricionais adicionais. Este estudo teve como objetivo avaliar as habilidades orais de crianças nascidas prematuras aos 4 e 6 meses de idade corrigida.

\section{MÉTODO}

Realizou-se um estudo longitudinal e prospectivo, no período de setembro de 2008 a abril de 2009, para avaliar as habilidades orais de 14 crianças nascidas com idade gestacional entre $29 \mathrm{e}$ 35 semanas, que permaneceram internadas na UTI Neonatal do Hospital Universitário de Santa Maria (HUSM), as quais foram acompanhadas no Ambulatório de Seguimento de Prematuros do mesmo hospital.

Foram incluídas na pesquisa, as crianças que receberam um APGAR no quinto minuto maior ou igual a 6 , sem evidências de problemas ou lesões neurológicas e cujos pais e ou representantes legais concordaram e assinaram o Termo de Consentimento Livre e Esclarecido. A presença de síndromes genéticas, malformações congênitas maiores e as crianças submetidas a tratamento fonoaudiológico, foram critérios de exclusão.

A avaliação das habilidades orais foi realizada quando as crianças tinham 4 e 6 meses de idade corrigida, tendo sido utilizado um protocolo (quadro 1) desenvolvido através de adaptação de vários estudos $^{4-9}$. Aos 4 meses de idade corrigida, a observação dos movimentos se deu através da sucção no seio materno ou no uso da mamadeira. Aos 6 meses, os movimentos avaliados foram através da mamadeira ou seio, da colher (tipo de chá, de aço inox, com alimento pastoso), do copo e da oferta de um pedaço de bolacha. O copo utilizado era de material transparente para permitir uma melhor visualização dos movimentos da língua e com borda recortada em forma de "U" de um lado, para acomodar o nariz. A borda recortada permitia que o copo fosse inclinado, de modo que todo o líquido pudesse ser consumido, evitando que a criança flexionasse a cabeça para trás ${ }^{10}$.

As mamadeiras, fornecidas pela pesquisadora, foram da marca Nuk@, com bico ortodôntico transparente de silicone, para as idade de 0 a 6 meses. Os alimentos ofertados e suas respectivas quantidades basearam-se no estudo de Telles e Macedo ${ }^{6}$, foram disponibilizados pela pesquisadora e todas as avaliações realizadas por fonoaudióloga.

Para avaliar, a criança era colocada no colo da mãe, que oferecia os alimentos, segundo as orientações da fonoaudióloga. No caso da utilização da bolacha, ela era introduzida na região lateral da boca, pela fonoaudióloga, observando-se a lateralização da língua e a mascagem. O tempo de duração da avaliação foi de no máximo quinze minutos. Todos os procedimentos foram filmados, com uma filmadora marca Sony DCR - SR $42 \AA$, sendo utilizado o mesmo enquadramento e distância em todas as avaliações, focalizando a região de lábios, mandíbula e língua.

A observação de cada movimento apresentado pela criança foi analisada, por 3 fonoaudiólogas (a que participou em tempo real das avaliações e duas externas) através de DVDs e anotada em ficha individual, conforme o quadro 1. A avaliação final de cada habilidade foi classificada em adequada (todos os movimentos e vedamento labial foram observados) e inadequada (não foram observados um ou mais movimentos e/ou vedamento labial). Todas as avaliadoras tinham experiência em motricidade oral.

Os pais informaram os hábitos alimentares da criança, através de um questionário que foi adaptado do estudo realizado por Pfitscher e Delgado ${ }^{11}$, com perguntas fechadas, de múltipla escolha sobre: tempo de aleitamento materno exclusivo, uso de complemento alimentar, a idade em que foram introduzidos os outros alimentos e qual a alimentação predominante, na época da avaliação.

O protocolo de investigação foi aprovado pelo Comitê de Ética em Pesquisa da Instituição sob o número 0131.0.243.000-06.

A análise dos dados foi feita através do software STATA ${ }^{12}$ utilizando estatística descritiva e a medida de tendência central moda, para definir a observação ou não de cada posição entre a avaliação das três fonoaudiólogas. Para avaliação da concordância entre as avaliadoras foi utilizado o Teste de Kappa. De acordo com a distribuição das variáveis estudadas, utilizou-se o teste t-Student, KruskalWallis e Exato de Fisher, sendo aceito como significante um valor de $p<0,05$. 


\section{Habilidade da sucção na mamadeira/seio aos 4 meses}

1. Movimento antero-posterior da língua durante a sucção nutritiva

Não observado ( ) Observado ( )

2. Movimento antero-posterior da mandíbula durante a sucção nutritiva

Não observado ( ) Observado ( )

3. Vedamento labial durante a sucção nutritiva

Não observado ( ) Observado ( )

Habilidade da sucção na mamadeira/seio aos 6 meses

1. Movimento elevação e abaixamento da língua durante a sucção nutritiva

Não observado ( ) Observado ( )

2. Movimento elevação e abaixamento da mandíbula durante a sucção nutritiva

Não observado ( ) Observado ( )

3.Vedamento labial durante a sucção nutritiva

Não observado ( ) Observado ( )

Habilidade da sucção com o uso do copo aos 6 meses

4. Movimento antero-posterior da língua ao utilizar o copo

Não observado ( ) Observado ( )

5. Mandíbula excursiona ao utilizar o copo

Não observado ( ) Observado ( )

6. Sorver o copo

Não observado ( ) Observado ( )

Habilidade de retirada do alimento da colher aos 6 meses

7. Lábio superior auxilia na retirada do alimento da colher

Não observado ( ) Observado ( )

Habilidade da lateralização da língua com o uso da bolacha aos 6 meses

8. Movimento da língua para o lado quando o alimento é introduzido pela lateral da boca Não observado ( ) Observado ( )

Habilidade da mascagem com bolacha

9. Movimento elevação e abaixamento da língua durante a mascagem

Não Observado ( ) Observado ( )

10. Início dos movimentos de lateralização da língua durante a mascagem

Não Observado ( ) Observado ( )

11. Movimento elevação e abaixamento da mandíbula durante a mascagem

Não Observado ( ) Observado ( )

Figura 1 - Protocolo de avaliação das habilidades orais

\section{RESULTADOS}

As habilidades orais de 14 crianças nascidas prematuras foram avaliadas aos 4 e 6 meses de idade corrigida. Ao nascimento, a média de idade gestacional foi de $31,6( \pm 2,0)$ semanas e a média de peso ao nascer de $1556( \pm 83,3)$ gramas. Das crianças avaliadas, $71,4 \%$ eram do sexo masculino e tinham, na primeira avaliação, uma média de 5,7 meses de idade cronológica e, na segunda avaliação, 7,7 meses. O aleitamento materno exclusivo foi verificado em 14,2\% (2/14) das crianças na avaliação dos 4 e 6 meses.

Em relação às habilidades orais, a concordância entre os avaliadores variou entre 60 a $100 \%$, dependendo dos movimentos, com exceção ao uso do copo, no qual a concordância foi de $31 \%$. Aos 4 meses, os movimentos da língua 
e mandíbula foram observados em $92,8 \%$ e $100 \%$ das crianças, respectivamente e o vedamento labial em $71,4 \%$. Aos 6 meses, o vedamento labial foi observado em $85,8 \%$ dos casos. Em relação ao uso da colher, em $78,6 \%$ das crianças, o lábio superior retirou de forma eficiente o alimento pastoso da colher. Quanto ao uso da bolacha, observou-se que $92,8 \%$ realizaram a lateralização da língua. Todas as crianças apresentaram elevação e abaixamento da língua e da mandíbula durante a mascagem. Em 92,8\% das crianças, o movimento da língua e mandíbula com o uso do copo foi observado, sendo que $42,9 \%$ foram capazes de sorver o líquido adequadamente, no entanto, a maioria, $57,1 \%$ não conseguiu sorver corretamente (Tabela 1).

Tabela 1 - Distribuição percentual dos movimentos de sucção, do vedamento labial e da das habilidades orais em crianças pre-termo aos 4 e 6 meses de idade corrigida

\begin{tabular}{lcc}
\hline Variáveis & Observado & Não Observado \\
& $\mathbf{N}(\%)$ & $\mathbf{N}(\%)$ \\
\hline Aos 4 meses & & \\
$\quad$ Movimento na sucção & $13(92,8)$ & $1(7,2)$ \\
$\quad$ Antero-posterior da língua & $14(100,0)$ & $0(0,0)$ \\
$\quad$ Antero-posterior da mandíbula & $10(71,4)$ & $4(28,6)$ \\
$\quad$ Vedamento labial & & \\
Aos 6 meses & & \\
$\quad$ Movimento na sucção & $13(92,8)$ & $1(7,2)$ \\
$\quad$ Elevação e abaixamento de língua & $14(100,0)$ & $0(0,0)$ \\
$\quad$ Elevação e abaixamento de mandíbula & $12(85,7)$ & $2(14,3)$ \\
$\quad$ Vedamento labial & & \\
Movimento no uso do copo & $13(92,8)$ & $1(7,2)$ \\
$\quad$ Antero-posterior da língua & $13(92,8)$ & $1(7,2)$ \\
$\quad$ Excursão da mandíbula & $6(42,9)$ & $8(57,1)$ \\
$\quad$ Sorver no copo & & \\
Preensão no uso da colher & $11(78,6)$ & $3(21,4)$ \\
$\quad$ Lábio superior na retirada do alimento & & \\
Movimento no uso da bolacha & $13(92,8)$ & $1(7,2)$ \\
$\quad$ Lateral da língua com o uso da bolacha & & \\
Movimento durante a mascagem & $13(92,8)$ & $1(7,2)$ \\
$\quad$ Lateralização da língua & $14(100,0)$ & $0(0,0)$ \\
$\quad$ Elevação e abaixamento da língua & $14(100,0)$ & $0(0,0)$ \\
$\quad$ Elevação e abaixamento da mandíbula & &
\end{tabular}

As habilidade orais estavam adequadas, aos 4 meses em 64,3\% das crianças. Aos 6 meses, foi adequada na sucção em $78,6 \%$, no uso da colher, em $78,6 \%$, no uso do copo, $64,3 \%$, na utilização da bolacha em $92,8 \%$ e, com todas as habilidades adequadas $14,3 \%$ das crianças (Tabela 2 ).

Em relação à concordância entre os avaliadores, verificou-se que, para a sucção, a mesma foi considerada muito boa $(k=0,65) \mathrm{com}$ a idade de 4 meses e perfeita $(k>0,80)$ aos 6 meses. No entanto, na avaliação da habilidade com o copo a concordância foi pobre $(k=0,31)$.

\section{DISCUSSÃO}

Estudos abordando o desenvolvimento das habilidades orais durante os primeiros meses são escassos na literatura, principalmente, em crianças prematuras. A avaliação das habilidades orais de 14 crianças que nasceram, prematuramente, aos 4 e 6 meses de idade corrigidas mostrou que a habilidade de sugar na mamadeira aos 4 meses, teve como dificuldade principal o vedamento labial, a qual diminuiu aos 6 meses. Na descrição do desenvolvimento normal das funções orais, o vedamento 
Tabela 2 - Habilidades orais de crianças pré-termo aos 4 e 6 meses de idade corrigida

\begin{tabular}{lcc}
\hline Variáveis & $\begin{array}{c}\text { Adequado } \\
\mathbf{N}(\%)\end{array}$ & $\begin{array}{c}\text { Inadequada } \\
\mathbf{N}(\%)\end{array}$ \\
\hline $\begin{array}{l}\text { Aos } 4 \text { meses } \\
\quad \text { Habilidade da sucção na mamadeira/seio }\end{array}$ & $9(64,3)$ & $5(35,7)$ \\
Aos 6 meses & & \\
$\quad$ Habilidade da sucção na mamadeira/seio & $11(78,6)$ & $3(21,4)$ \\
$\quad$ Habilidade da sucção com o uso do copo & $5(35,7)$ & $9(64,3)$ \\
$\quad$ Habilidade de retirada do alimento da colher & $11(78,6)$ & $3(21,4)$ \\
$\quad$ Habilidade da lateralização da língua com o uso da bolacha & $13(92,8)$ & $1(7,2)$ \\
$\quad$ Habilidade da mascagem com o uso da bolacha & $13(92,8)$ & $1(7,2)$ \\
$\quad$ Todas as habilidades & $2(14,3)$ & $12(85,7)$ \\
\hline
\end{tabular}

$\mathrm{N}$ : número de crianças.

$\%$ : porcentagem de crianças.

labial é considerado como um comportamento normal da sucção em lactentes ${ }^{8}$. Este fato também foi observado por Delgado e Halpern ${ }^{13}$ ao avaliarem crianças nascidas prematuras com peso ao nascimento menor que $1500 \mathrm{~g}$ em que o vedamento labial foi inadequada em $76,5 \%$ delas. Deve ser levado em conta que o recém nascido prematuro apresenta um comprometimento da força, assim como da resistência durante a sucção, que pode se estender aos primeiros meses de vida ${ }^{14}$. Esses fatores podem justificar a dificuldade em realizar o vedamento labial na população do presente estudo, pelo menos em parte, uma vez que outros fatores como a hipotonia muscular e o desenvolvimento do tônus muscular flexor, que se faz de maneira caudal-cranial, podem também ter contribuído. É importante salientar que o tônus muscular pode não se desenvolver com a mesma eficiência observada na criança nascida a termo ${ }^{15}$.

Aos 6 meses de idade corrigida, ao serem avaliadas as habilidades orais, com exceção da sucção, observou-se que o movimento de retirada do alimento pastoso da colher, pelo lábio superior, não foi eficiente em todas as crianças. No entanto, este seria um movimento esperado em uma criança com 6 meses de idade cronológica9 ${ }^{\text {. Dificuldades }}$ nas primeiras tentativas, em usar a colher, para ingerir alimentos pastosos foram descritas por Marujo $^{3}$, porém, ressalta-se que as crianças deste estudo, já tinham feito a transição alimentar, em média há 1,6 meses. Assim, as dificuldades observadas não poderiam ser atribuídas às primeiras tentativas do uso da colher, o que aponta para a necessidade de se monitorar o desenvolvimento oral de prematuros.

Em relação aos movimentos de mandíbula e língua, com o uso da bolacha e na mascagem, na maioria das crianças, não foram observadas dificuldades aos 6 meses de idade corrigida, fato esperado uma vez que a criança, nessa idade, tem capacidade para mover sua língua lateralmente, buscando o alimento que é colocado do lado da boca $^{9}$. No entanto, em estudo com crianças a termo, Telles e Macedo 6 , verificaram prejuízo nas habilidades referentes à mascagem aos 6 meses, sendo $31 \%$ nos movimentos de língua e $24 \%$ nos movimentos de mandíbula. Ressalta-se que o estudo não tinha como objetivo analisar os hábitos alimentares familiares que podem influenciar na aquisição da mascagem ${ }^{11}$.

O uso do copo é um método alternativo em casos de fracasso do aleitamento materno ${ }^{16}$. Nas crianças avaliadas, embora com desmame precoce, $57,1 \%$ não haviam utilizado o copo aos 6 meses de idade corrigida e $57,1 \%$ das crianças não foram capazes de sorver de maneira adequada o líquido do copo. Dificuldades semelhantes foram relatadas em um estudo de Costa Alves et $\mathrm{al}^{4}$, realizado com 27 lactentes nascidos a termo, com idades entre 6 a 12 meses, onde apenas $18,5 \%$ dos lactentes estavam aptos para utilizar o copo de forma adequada. A dificuldade, com o uso do copo aos 6 meses de idade, provavelmente demonstre o pouco uso do mesmo em crianças no primeiro ano de vida, podendo ainda ser reflexo dos costumes familiares para a utilização de certos alimentos, uma vez que já foi demonstrado, por exemplo, a importância dos hábitos alimentares da família na aquisição da mascagem ${ }^{11}$. A avaliação da habilidade, no uso do copo, também determinou uma das limitações deste estudo que foi a concordância insatisfatória entre os avaliadores. 


\section{CONCLUSÃO}

Conclui-se com este estudo que o desenvolvimento das habilidades orais em recém-nascidos pré-termo se faz de forma sequencial e progressiva de modo que, aos 6 meses de idade corrigida, a sucção, a retirada do alimento da colher, a lateralização da língua e a mascagem estão presentes na maioria das crianças. Já a habilidade para sorver o líquido do copo não está completa aos 6 meses, sugerindo a necessidade de estimulação para uma melhora desta performance.

\begin{abstract}
Purpose: to evaluate the oral skills of preterm infants at 4 and 6 months of corrected age. Methods: 14 preterm infants born between 29 and 35 weeks of gestational age were evaluated during their follow-up at the University Hospital of Santa Maria, state of Rio Grande do Sul. Assessment at 4 and 6 months of corrected age included movements of the tongue, lips and jaw during bottle or breastfeeding, and at 6 months, we also assessed their ability to remove the pasty food from the spoon, the use of the cup, and with munching biscuits. All assessments were recorded and analyzed by 3 speech therapists. The parents answered a questionnaire on the dietary habits of their children. Results: at 4 and 6 months of corrected age an appropriate lip sealing was observed in $71.4 \%$ and $85.7 \%$ of the infants, respectively. At 6 months, in $78.6 \%$ of them the upper lip removed efficiently the pasty food from the spoon, $42.9 \%$ were able to absorb the liquid from the cup and $57.1 \%$ had difficulties to do this well. Conclusion: in this study, infants born preterm at 4 and 6 months of corrected age showed the development of oral skills in a progressive sequence, although they showed a compromised lip sealing during sucking and difficulties in using spoon and cup for their feeding.
\end{abstract}

Keywords: Child; Feeding Methods; Infant, Premature

\section{REFERÊNCIAS BIBLIOGRÁFICAS}

1.Barlow SM, Estep M. Central pattern generation and the motor infrastrucuture for suck, respiration, and speech. Journal of Communication Disorders. 2006; 39: 366-80.

2. Boiron M, Roux S, Henrot A. Effects of oral stimulation and oral support on non-nutritive sucking and feeding performance in preterm infants. Developmental Medicine \& Child Neurology. 2007; 49:439-44.

3. Marujo VLB. Fonoaudiologia em paralisia cerebral. In: Souza, AMC, Ferraretto, I. (org.) Paralisia cerebral: aspectos práticos. São Paulo. Memnon. 1998. p. 207- 30.

4. Alves CA, De Araújo CV, Guedes ZCF. Habilidade na utilização dos utensílios copo e canudo por lactentes de 6 a 12 meses. Fono Atual, 2005; 33(8): 4-10.

5. Rocha AD, Moreira MEL, Pimenta HP, Ramos JRM, Lucena SL. A randomized study of the efficacy of sensory - motor - oral stimulation and non nutritive sucking in very low birthweight infant. Early Human Development. 2007; 83 (6):385-8.
6. Telles SM, Macedo CS. Relação entre desenvolvimento motor corporal e aquisição de habilidades orais. Pró-Fono Revista de Atualização Científica. 2008; 20(2):117-22.

7. Pridhman KF, Stewaed D, Thoyre S, Brown R, Brown L. Feeding skill performance in premature infants during the first year. Early Human Development. 2007; 83:293-305.

8. Howe T, Sheu C, Hinojosa J, Lin J, Holzman I. Multiple factors related to bottle-feeding performance in preterm infants. Nursing Research. 2007; 56 (5): 307-11.

9. Carruth BR, Skinner JD. Feeding behaviors and other motor development in healthy children (2-24 months). Journal of the American College of Nutrition. 2002; 21(2): 88-96.

10. Mueller HA. Alimentação. In: Finnie NR. O manuseio em casa da criança com paralisia cerebral. São Paulo. Manole. 2000.p. 226.

11. Pfitscher AP, Delgado SE. A caracterização do sistema estomatognático, após a transição alimentar, em crianças prematuras de muito baixo peso. Rev. Soc. Bras. Fonoaudiol. 2006; 11(4): 215-22.

12. Stata Versão 10. Stata press publication. College Station Texas: Stata Corpe LP. 2007. 325 p. 
13. Delgado SE, Halpern R. Amamentação de prematuros com menos de 1500 gramas: funcionamento motor oral e apego. Pró-Fono Revista de Atualização Científica. Barueri (SP). 2005; 17(2):141-52.

14. Lau C, Alagugurusamy $Y R$, Schanler RJ, Smith EO, Schulman RJ. Characterzation of the developmental stages of sucking in preterm infants during bottle feeding. Acta Paediatr. 2000; 89: 846-52.
15. Mello RS, Da Silva KS, De Rodrigues MCC, Chalfun G, Ferreira RC, Delamônica JVR. Predective factors for neuromotor abnormalities at the corrected age of 12 months in very low birth weight premature infants. Arq. Neuropsiquiatr. 2009; 67 (2-A): 235-41.

16. Lima VP, Melo AM. Uso do copinho no alojamento canguru. Rev. CEFAC. 2008; 10 (1):126-33.

doi: 10.1590/S1516-18462010005000098

RECEBIDO EM: 01/02/2010

ACEITO EM: 23/04/2010

Endereço para correspondencia:

Aneline Maria Ruedell

Rua Carlos de Carvalho, 3496 apt. 23

Cascavel - PR

CEP: 85801130

E-mail: aneruedell@yahoo.com.br 